\title{
Use of geographical information systems for delimiting health service areas in China
}

\author{
Xuechen Xiong, Li Luo \\ School of Public Health, Fudan University, Shanghai, China
}

\begin{abstract}
With the objective of choosing a practical and valid method to delimit health service areas of regional health service centres to build a regional basic health service network, we first drew lessons from traditional geographic methods of delimiting trade areas and then applied two methods to delimit health service areas, i.e. the proximal method and the gravity method. We verified the effectiveness of these methods by an index of similarity with the aid of real in-patient data. Calculation of the similarity indices shows that health service areas delimited by the proximal method has an $87.3 \%$ similarity to the real health service area, while the gravity method gives $88.6 \%$. Our conclusion is that both methods
\end{abstract}

Correspondence: Li Luo, School of Public Health, Fudan University, 220 Handan Rd., Shanghai, 200433 China.

Tel: +8613641752131 .

E-mail: 1iluo@fudan.edu.cn

Key words: Health service area; Basic health service; Regional health planning; GIS; Chinese health system reform.

Contributions: all authors contributed to the design of the study. LL and XX conceived designed and implemented the analysis including exploratory data analysis and visualisation of data and data processing. XX collected and analysed the research data and drafted the manuscript. LL revised the paper. LL and XX read and approved the final manuscript.

Funding: funding for this research was provided by the National Natural Science Foundation of China (No. 71473045) and the Key Projects of Philosophy and Social Sciences Research, Ministry of Education, China (No.15JZD029).

Conflict of interest: the authors declare no potential conflict of interest.

Received for publication: 20 April 2016.

Revision received: 21 October 20016.

Accepted for publication: 1 March 2017.

CCopyright X. Xiong and L. Luo 2017

Licensee PAGEPress, Italy

Geospatial Health 2017; 12:486

doi:10.4081/gh.2017.486

This article is distributed under the terms of the Creative Commons Attribution Noncommercial License (CC BY-NC 4.0) which permits any noncommercial use, distribution, and reproduction in any medium, provided the original author(s) and source are credited. are suitable for delimiting health service areas at regional health service centres, but find that the proximal method is more practicable in operational terms for delimiting health service areas in region health planning.

\section{Introduction}

\section{Chinese regional health planning}

In the past decade, China's health care sector has made remarkable progress. However, we have to recognise that the current development level of China's health care sector does not fully meet people's health demands (Yip and Hsiao, 2008). In order to improve the system, a new reform was launched in 2009 (National Development and Reform Commission, 2009). The overall goal of this new approach is to establish and improve the basic health care system covering urban and rural residents alike and provide people with secure, efficient, convenient and affordable health care service. In order to achieve this goal, the Chinese Government aims to establish a rationally structured health care service system that can deliver different kinds of health service and health product to satisfy all various health demands.

The Chinese health care structure consists of three tiers (Hsiao, 1995; Rongwen, 2011; Tao et al., 2012), the first of which is a grass root medical service network combined with township health centres and village clinics. It serves as a base-level public health service providing diagnosis and treatment of common, uncomplicated diseases (Shi, 2012). The second tier consists of district-level hospitals, many of which are situated in the rural areas. The responsibility of these hospitals is to provide developed health care through provision of bed facilities and treatment of patients with severe or acute diseases. The third tier consists of teaching hospitals and research centres that serve as bellwether organisations, the main goal of which is to deliver advanced health care service, including treatment of unusual and difficult diseases that require specialist treatment. In theory, the health care system should perform efficiently. However, as it is free for a patient to choose whichever hospital in his/her city of residence, the ability of the health medical insurance authority to distribute patients is limited. Under this circumstances, patients tend to seek medical service from advanced hospitals even when it is not necessary, which results in overcrowding. Meanwhile, the district-level regional hospitals accept much fewer patients than expected. This situation reflects the potential slack in the system, i.e. the first- and second-tiers of health care service system do not get the opportunity to play their role in the system. This runs counter to the theory of ecology of medical care as proposed by White (1997) and discussed by Leung et al. (2005). In summary, the most challenging 
part of the current health care service system in China is the coexistence of excessive demand for advanced medical service and the idle basic health care service (Zhao, 2010). How to activate the latter guaranteeing a rational use of the medical capabilities at each level has become a crucial problem that urgently needs to be solved.

\section{The regional health service centre}

The Chinese Government realises the importance of adjusting the utilisation of the health service system. In recent years, it has made many efforts to distribute patients rationally through administrative steps, e.g., there is continuing strong investment in basic healthcare to encourage district-level hospitals in the countryside to improve their technology level (Barber, 2014). Shanghai, serves as the forefront in intensifying health care system reform and has put forward the new concept of a regional health service centre (Zhou, 2009; Ge et al., 2013; Zhu, 2014). Functionally, this centre is similar to a district-level hospital since both provide basic health services. However, the most striking difference between these concepts is that the regional health service centre emphasises the health service area, i.e. it clarifies and defines the responsibility with special reference to the geographical area it serves. This implies that the regional health service centre is listed as the leading hospital that should undertake the obligation of basic health care service in the area in question.

Shanghai is building a two-tier health care service, the first of which combines regional health service centres, township health centres and village clinics, a scheme located in the centre's health service area that provides basic health care. The second tier combines teaching hospitals and research centres into a element aspiring to be a bellwether by delivering high-level health care as well as tending to patients with diseases that are difficult to treat. The regional health centre of the first tier thus aims to deliver basic health service for all its residents.

Regional health planning aims to provide every person with secure, efficient, convenient and affordable health care (Shen, 2013; Yao, 2015). The Government can adjust and relocate health resources to needed areas, but to achieve the goal stated, there are at least two questions that need to be answered. What areas are lacking health resources and what is the shortfall of these resources? The first question concerns the geographic units used as the geo-cell to evaluate whether the health resource in each specific area is sufficient. The second question concerns the criterion to evaluate whether the health resource of a certain region is sufficient. Our aim concerns primarily the first question. The Chinese Government uses a crude administrative boundary as the geographic units to allocate health resources, such as the district unit. However, health-seeking behaviour does not always match the political boundary. In this paper we are trying to break away from the limitation of administrative boundaries to make sure the health service areas can correspond to the real situation where healthseeking behaviour plays an important role. In the two-tier system, the teaching hospitals and research centres serve the whole city, while regional health service centres serve only parts of the city (here called the health service area). The next step is to find out where the health service area of the regional health service centre is located and how to delimit the service offered. This is the key technical question that has to be answered in a regional health plan, and this paper is trying to choose a proper method to do so by building a basic health service geographic network.

\section{Delimiting the health service area}

There are three main methods to delimit trade areas: the analogy method (Applebaum, 1965, 1966; Erickson and Finkler, 1985), the proximal area method (Okabe et al., 2008; Peipins, 2013; Guerrero and Kao, 2013) and the gravity method (Huff, $1963,1964)$. The analogy method is non-geographic and often implemented by regression analysis as first proposed by Applebaum $(1965,1966)$. This approach has subsequently been developed to determine the levels of market penetration using stores and shopping as example (Olsen and Lord, 1979). This analogy is easy to implement, but it has major weaknesses. On the one hand, stores are selected subjectively without considering the situational and site characteristics that can be presumed to affect their performance. In addition, although the data may be used to plot market penetration at various distances from the store, the major objective is to forecast sales, not to define trade areas geographically (Wang, 2014). The proximal method and the gravity model are both geographic approaches and can be complemented by geographical information systems (GIS) technology. The proximal method is performed under the assumption that consumers choose the nearest store among similar outlets (Ghosh and McLafferty, 1987), which implies that travel distance (or travel time) is the only factor that affects customer choice. In this scenario, the trade area is simply made of consumers that are closer to the store than others. However, consumers sometimes bypass the closest store to shop in stores with better prices, better goods, larger assortments or better general image (Wang, 2006). Unlike the proximal method, the gravity model considers distance (or time) and the relative attractiveness of various stores. In the early days, Reilly's law (1931) of retail gravitation applied the concept of a gravity to delineate trade areas. However, this law only defines trade areas between two stores. A more general method using the gravity model is the Huff approach, which defines trade areas for multiple stores (Garnick et al., 1987). According to Huff (2003), the model's widespread use and longevity can be attributed to its comprehensibility, relative ease of use and its applicability to a wide range of problems.

In addition, there is a large body of literature of delineating health (hospital) service areas using other methods (Klauss et al., 2005; Berlin, 2014). Foremost, the Dartmouth method (Dartmouth Institute for Policy and Clinical Practice, 1998) used the Medicare patient flow data to delineate health service areas that hold most flows within the health service areas. This method is based on the real health-seeking behaviour of patients and, as a result, the delineated health service areas are close to the realistic health service area. With regard to effectiveness, the Dartmouth method is better for delineating health service area. However, too much data are needed for this approach, and it is difficult to collect complete sets of such data, especially in developing countries. In this paper, we try to find a universal and practicable method to delimit health service area for regional health service centres rather than analyse the patient flow or delineate the health service areas for a certain region. We consider therefore the realistic health service areas, based on the real health-seeking behaviour of patients as the 'gold standard' that validates the efficiency of other health service delimiting methods.

\section{Overall purpose}

The purpose of this paper is to choose a practical and valid method to delimit the health service areas of regional health service centres to guarantee basic health care service for all residents 
through building a geographic regional health-planning network. The health service areas are delimited as the geographic unit to evaluate whether it is in shortage of basic health resources and if so available adjust health resources. The research design is demonstrated for the case of Shanghai with the following framework with the idea to: i) learn from the methods for trade area analysis focusing on geographic approaches to specify health service areas using the proximal and the gravity method; ii) use available data on health-seeking behaviour of residents to verify the reliability and validity of the delimited health service areas using the proximal and the gravity method; and iii) draw a conclusion based on comparison of the results of these two methods.

\section{Materials and Methods}

\section{The proximal method}

With regard to the level and scale of hospitals, 26 regionallevel hospitals were chosen to investigate how to delimit health service areas; this approach is based on the assumption that these hospitals mainly serve local residents and that residents prefer to access the nearest hospital. According to this rule, the health service area of each hospital would be composed of residents closer to this hospital than to any of the other 25 hospitals (Table 1).

The proximal method can be implemented in GIS to delimit health service areas in two ways. The first approach is consumerbased, and it begins with the location of a resident and searches for the nearest hospital using all hospital locations. The process continues until all resident locations are covered. At the end, residents who share the same nearest hospital constitute the proximal area for that hospital. The second approach is store-based and relies on the construction of Thiessen polygons (Wang, 2014) using the hospital locations, with the polygons around each hospital defining the proximal area for that hospital. The Thiessen polygons depend on the spatial distribution of the hospitals, and they may not cover all resident locations. Therefore, we completely delimited the health service areas using the proximal method described in the first approach in the following steps. A point layer of regional hospitals and resident locations (Figure 1) was constructed.

Secondly, based on the road network of the city, the road network distance from the location of each resident to all 26 hospitals was calculated. Thirdly, for every resident location, the nearest hospital among all hospital locations was identified. Finally, the residents sharing the same hospital constitute the proximal area for that hospital. Figure 2 illustrates the combination of resident locations and Figure 3 shows the health service areas drawn using the proximal method. All these calculations were performed in ArcGIS 10.0 (http://www.esri.com/software/arcgis).

\section{The gravity method}

The proximal area method considers distance (or time) only. However, consumers frequently bypass the nearest store to enter other stores with better goods, better prices or a better image. The same behaviour also occurs with hospitals because distance (or time) is not the only deciding factor. The gravity method considers two variables: distance (or time) and the attraction of the store. The Huff model of the gravity method as applied delimitation of trade areas is based on the following equation:

$$
\mathrm{P}_{i j}=S_{j} d_{i j}^{-\beta} / \sum_{k=1}^{n}\left(S_{K} d_{i k}^{-\beta}\right)
$$

where $\mathrm{P}_{i j}$ is the probability of an individual $i$ selecting a hospital $j$; $K$, (i.e. $k=1,2, \ldots, \mathrm{n}$ ) the alternative hospitals available; $S$ the hospital size (i.e. the number of beds); $d$ the distance; and $b$ the distance friction coefficient. The term $S_{j} d_{i j}{ }^{-\beta}$ is also referred to as potential, which measures the impact of a hospital $j$ on a demand location $i$. Equation 1 shows that the probability $\mathrm{P}_{i j}$ is positively related to the hospital size and inversely related to the distance between the hospital and a patient's residence. On the basis of this equation, residents in a region visit different hospitals with varying probabilities, and the health service area of a hospital is defined as the area with the highest probability of being visited. In practice, given a resident's location ( $i$, the denominator in this equation is identical for various hospitals $(j)$ and thus, the highest value of the numerator identifies the hospital with the highest probability of being visited. In other words, the needs of residents identify the hospital with the highest potential for defining the health service area, a design that can be implemented in ArcGIS.

With the exception of $\beta$, the distance friction coefficient, all

Table 1. Count of hospital beds of twenty-six health centres.

\begin{tabular}{|c|c|}
\hline Regional health service centre & Beds (n) \\
\hline 1 & 1050 \\
\hline 2 & 805 \\
\hline 3 & 800 \\
\hline 4 & 730 \\
\hline 5 & 700 \\
\hline 6 & 502 \\
\hline 7 & 630 \\
\hline 8 & 790 \\
\hline 9 & 830 \\
\hline 10 & 600 \\
\hline 11 & 600 \\
\hline 12 & 350 \\
\hline 13 & 600 \\
\hline 14 & 325 \\
\hline 15 & 600 \\
\hline 16 & 671 \\
\hline 17 & 800 \\
\hline 18 & 1000 \\
\hline 19 & 360 \\
\hline 20 & 800 \\
\hline 21 & 350 \\
\hline 22 & 527 \\
\hline 23 & 300 \\
\hline 24 & 201 \\
\hline 25 & 500 \\
\hline 26 & 425 \\
\hline
\end{tabular}




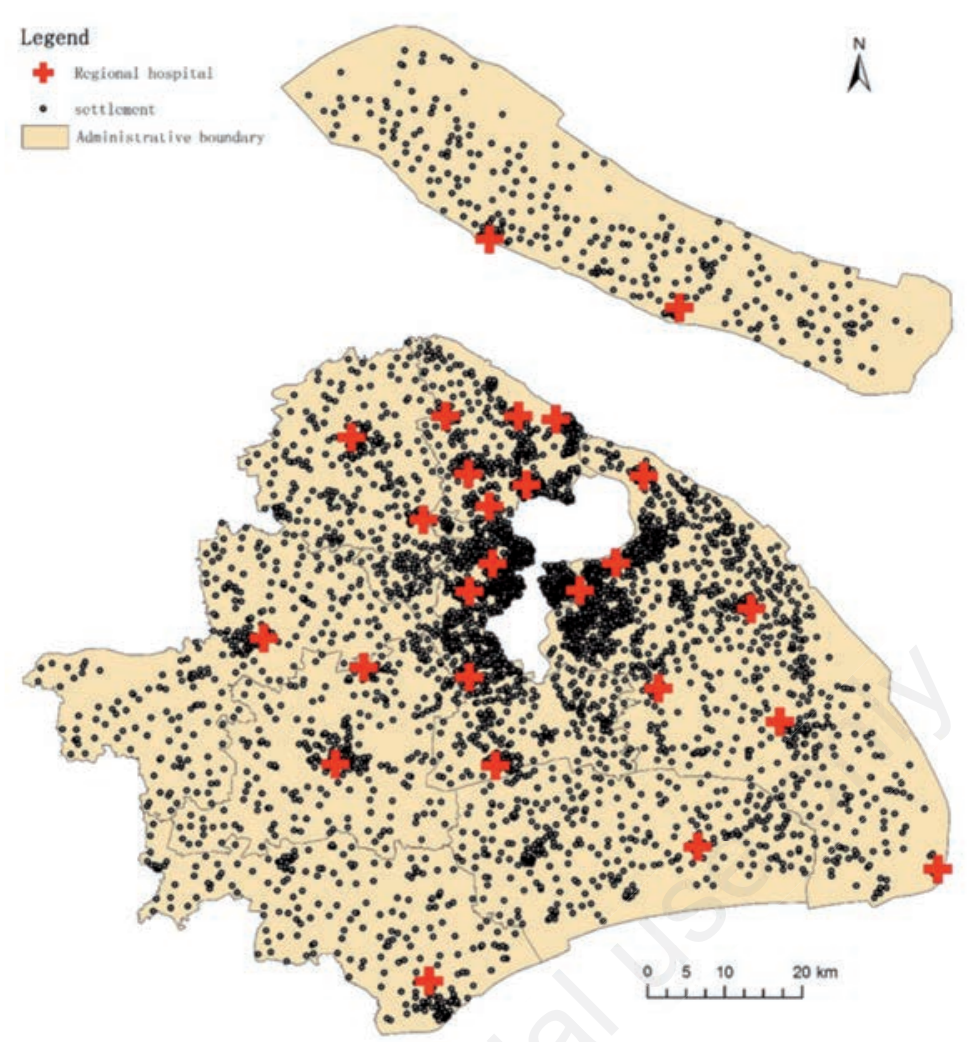

Figure 1. The distribution of regional hospitals and residents in Shanghai.

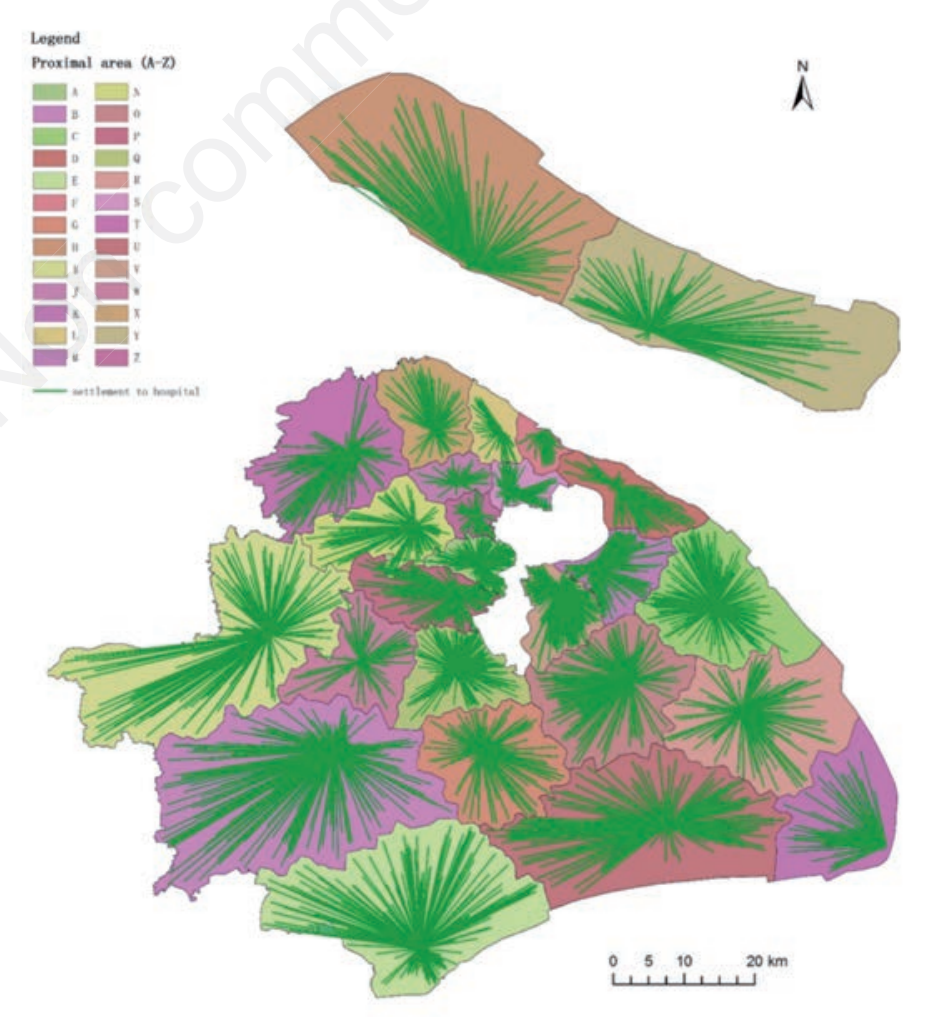

Figure 2. Combination of resident locations in Shanghai. 
parameters in the formula can be obtained from hospital data and road network data. In the gravity model, $\beta$ plays the key role, so it is necessary to derive the value of $\beta$ prior to the usage of the gravity model. A few studies have discussed methods of estimating the parameter $\beta$ (e.g. Jin et al., 1980). However, as no common method has been put forward to derive $\beta$, let alone a universal value of $\beta$, to be used in the gravity model we assumed $\beta=2$ in this paper.

The operational procedure for delimiting health service areas can be described by first creating a point-layer of regional hospitals and resident locations (Figure 1). Secondly, based on the road network of the city, one calculates a distance matrix between each resident and each hospital location, i.e. $d_{i j}$. Thirdly, based on the distance matrix, one counts the number of beds at every hospital $\left(S_{j}\right)$ and calculates the value of $\mathrm{P}_{i j}\left(\right.$ or $\left.S_{j} d_{i j}{ }^{-2}\right)$. Finally, given the location of each resident, one chooses the hospital with the highest visitation probability. In this way, and all the resident locations with the same hospital define the health service area (Figure 4).

\section{Definition of the real health service area: the gold standard}

Health service areas have been delimited for regional health planning both by the proximal and the gravity method to find out which one is the more valid one and which would be the most general one for promotion in other regions. To answer this question, we obtained the in-patient database of a sample city from the Health Bureau with the aim to illustrate the in-patient distribution of every hospital based on real in-patient data thus creating the foundation of the real health service areas in a region. Our approach to validate the effectiveness of the two methods in delimiting the health service area consisted of calculating the real health service areas in a region using the in-patient data as the gold standard. All calculations were implemented in ArcGIS 10.0 and SAS 9.2 (http://support.sas.com/documentation/92/) and the following operative steps were applied.

First, draw an in-patient scatter plot for each hospital (Figure 5). Second, together with the population distribution data, calculate the hospitalised probability of patients for each hospital, then simulate a continuous probability surface using the Inverse Distance Weighted method (Wang, 2014) (see Figure 6 as an example of the continuous hospitalised probability surface of hospital A). The probability here refers to the probability of an in-patient who lives in the region to seek health service in hospital A. The higher the probability, the more attractive the hospital is. Third, divide the map of Shanghai into $1000 \mathrm{~m}^{2}$ squares and extract the centroid of each square as the probability observation site. Fourth, determine the value of the probability at the probability observation site. Fifth, overlay the probability of all 26 hospitals. In this way, every probability observation site has 26 probabilities, one for each hospital. Sixth, extract the highest probability among all 26 hospitals for every probability observation site and deliver the highest probability to the square where the observation site lies. Seventh, the health service areas can now be specified by assigning each square to the health service area of the hospital that is visited with the highest probability. Figure 7 shows the result of analysis of the real health service areas.

\section{Validation}

The real health service areas calculated in this way were used as the 'gold standard' to test the ability of the two methods to delimit the health service areas. The next question that needed to be answered is how to use the real health service area to validate the reliability of the health service areas delimited by each method. In practice, an index of similarity that could be calculated in ArcGIS 10.0 was used as a testing index to verify the reliability of the approach investigated. The specific steps used to calculate this index were as follows. First, make a buffer zone for the boundaries of the real health service areas. Since these boundaries are not exact, neither in reality nor in the calculated model, the buffer zones work as overlap areas, in which the location of some residents can fall into various service areas (however, mainly no more than two); Second, divide the map of Shanghai into $500 \mathrm{~m}^{2}$ squares and extract the centroid of each as the property observation site. Third, overlapping health service areas are delimited by the proximal method (Figure 3)/the gravity method (Figure 4) and the real health service areas (Figure 7). The spatial relationship between the layers of these two health service areas and the property observation sites are thus established. Fourth, based on the spatial relationship, deliver the properties of the health service areas to the property observation. This step can be completed using the spatial join tool in ArcGIS. As a result, every property observation has two properties from the health service areas; one is from the real health service area and the other from the health service area defined by the proximal method/the gravity method. Fifth, for every property observation site, compare the two properties. If they differ, the result suggests that the health service area where the property observation is located is different from the realistic health service area when delimited by the proximal method/the gravity method. Otherwise, the result suggests that the properties of these two health service areas are the same. Therefore, the value of the similarity index is determined by dividing all the property observation points by the property observation point with the same property.

\section{Results}

The proximal method and the gravity method were both used to delimit health service areas for the purpose of this paper, and they each divided a region into several areas to minimise differences among planning regions. The results of these geographic methods are shown in Figures 3 and 4.

In addition, to verify the validity of these methods in delimiting health service areas, practical inpatient data have been analysed to simulate the realistic health service areas as the gold standard. The indexes of similarity are put forward as the test indexes. The similarity indexes show that the health service areas delimited by the proximal method had a similarity of $87.3 \%$ with the realistic health service areas, while delimitation using the gravity method had a the corresponding similarity of $88.6 \%$. At the same time, the different areas of the verification map were primarily located in the boundaries of the health service areas. It seems thus that the proximal method and the gravity method are almost equally good at delimiting health service areas though the gravity method is slightly better in this respect.

\section{Discussion}

The innovation of this paper is that we draw lessons from traditional geographic methods of delimitating trade areas and apply these method in a health field to define health service areas for regional health planning. In addition, with the help of realistic 


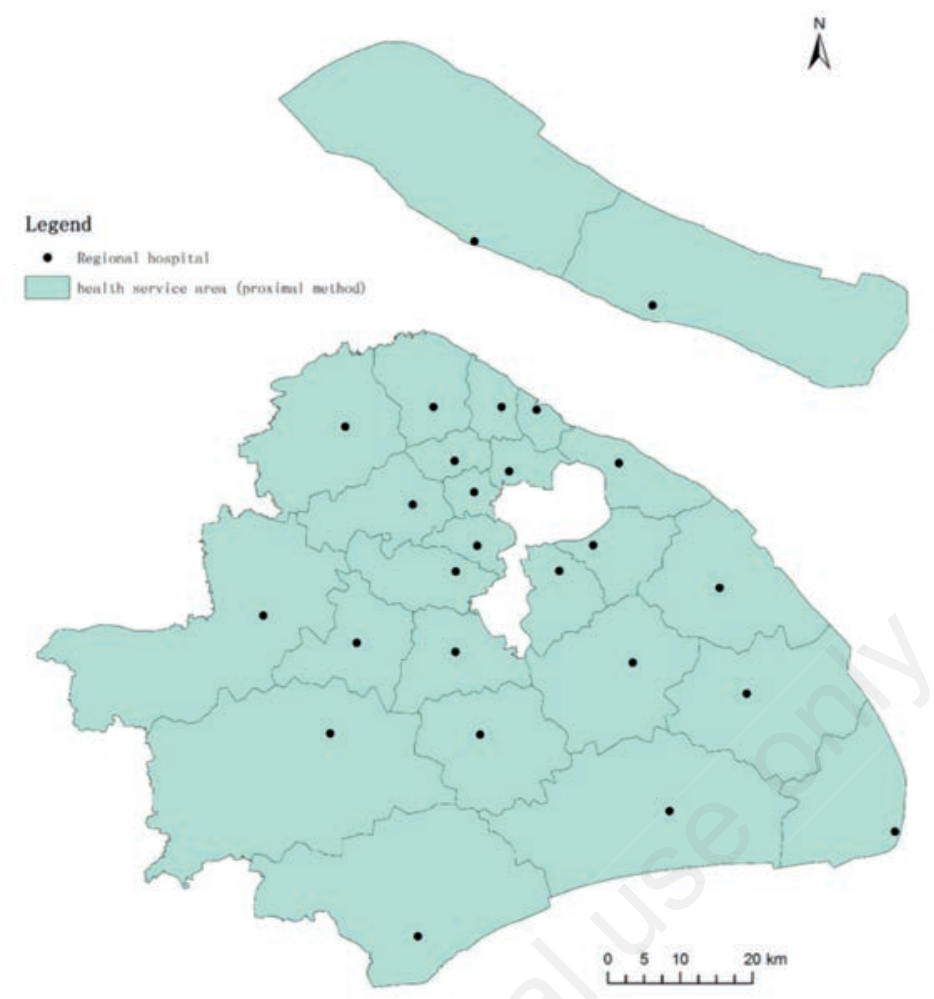

Figure 3. Health service areas delimited by the proximal method in Shanghai.

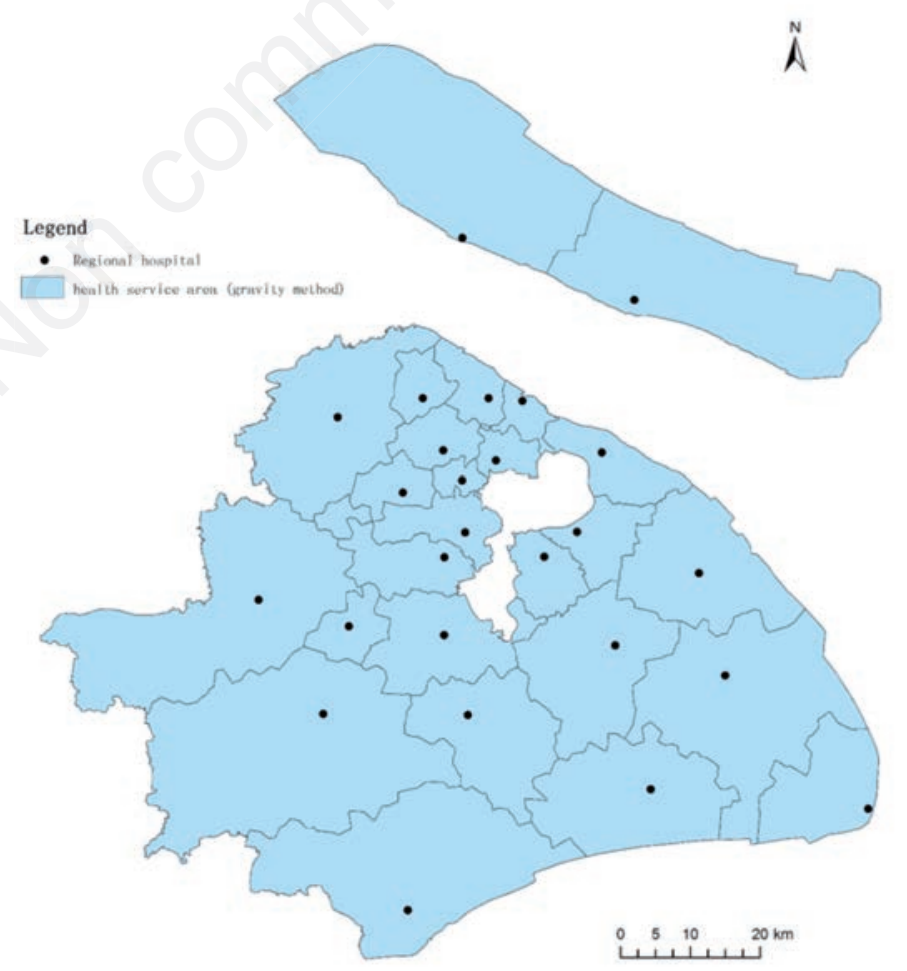

Figure 4. Health service areas delimited by the gravity method in Shanghai. 


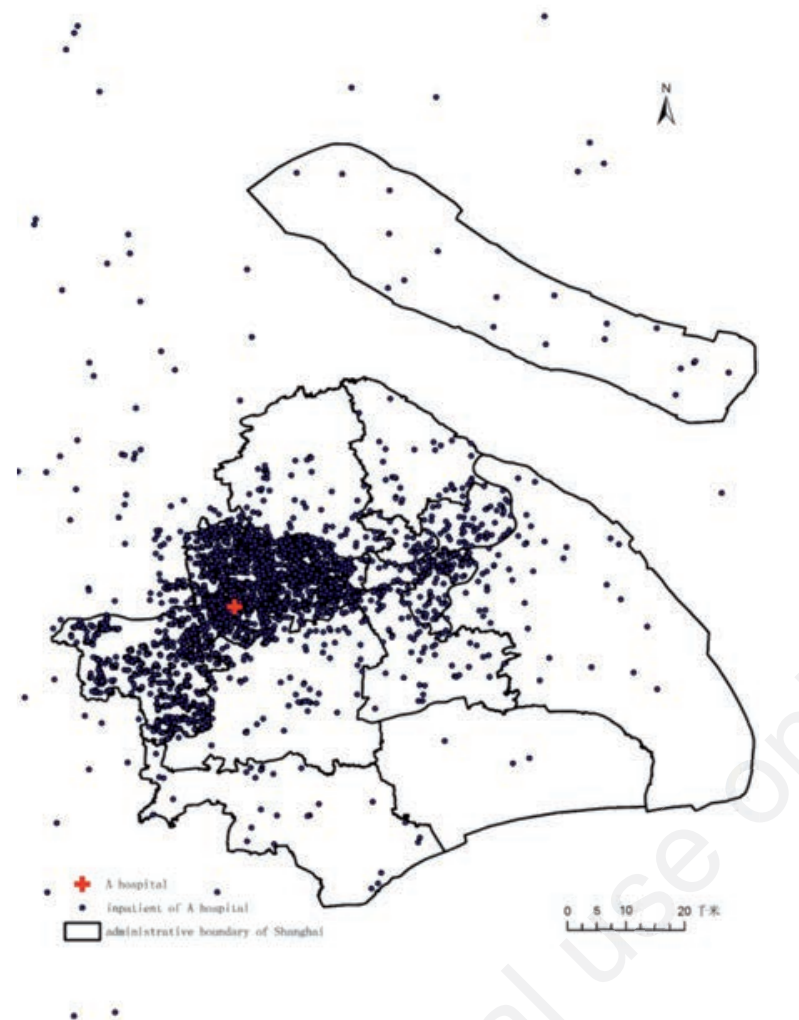

Figure 5. In-patient distribution of hospital A in Shanghai.

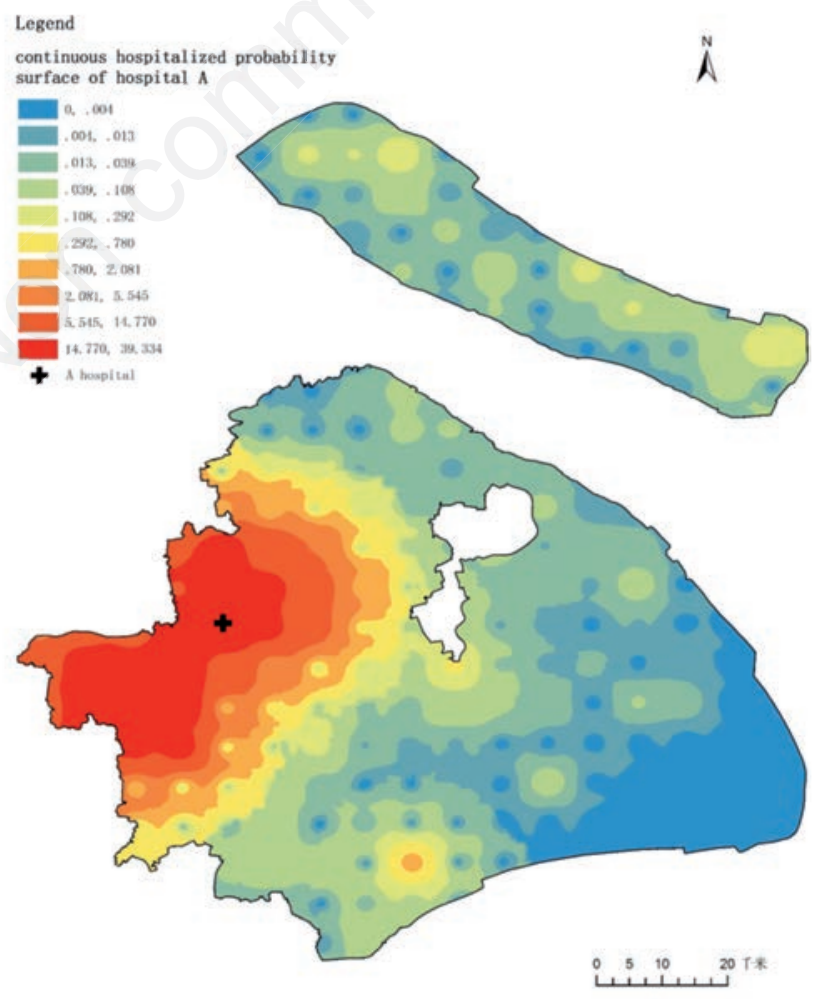

Figure 6. Continuous hospitalised probability surface of hospital A in Shanghai. 
inpatient hospitalised data, the methods used to delimit the health service areas are verified to make sure they are valid and efficient.

The method of delimiting health service areas of regional health service centres by the proximal method has been accepted by the Health Bureau of Shanghai. It has been applied in Shanghai's regional health planning (2015-2020) where it plays an important role for guaranteeing basic health care services to all residents. The delimitation of health service areas embodies the responsibility of providing basic health services in terms of geographical distribution giving the government a list of prior hospitals when investing in the development of basic health care services. What is more, the health service areas break the limitation of administration boundary. In fact, the boundary of health service areas is more appropriate than the administration boundary in regional health planning because the administration boundary is not the main factor affecting patients' health seeking behaviour, as was demonstrated by the realistic health service area. Therefore, in practice, the boundary of health service areas will take place of the administration boundary in regional health planning.

In this paper, there are several key difficulties that need to be overcome in the process of delimiting health service areas.

First, out of the three main methods to delimit trade areas (the analogy method, the proximal area method and the gravity method), we focused on the two latter approaches, i.e. the geographic methods. It is worth noting that these two methods can be implemented in GIS to delimit health service areas in other ways. For example, the original Huff model used in this study does not include an exponent associated with the hospital scale $(S)$. A sim- ple improvement over the Huff model is expressed as:

$\mathrm{P}_{i j}=S_{j}^{\alpha} d_{i j}^{-\beta} / \sum_{k=1}^{n}\left(S_{K} d_{i k}^{-\beta}\right)$

where the exponent $\alpha$ captures the elasticity of hospital size (Wang, 2006). For example, a larger hospital tends to be more attractive than its size alone suggests because of economy of scale. The improved model still only uses size to measure the attractiveness of a store. Nakanishi and Cooper (1974) proposed a more general form called the multiplicative competitive interaction model. In addition to size and distance, this model accounts for factors such as store image, geographic accessibility, and other store characteristics. All of these extensions of the Huff model are more accurate than the general Huff model, but they require more data to define the trade areas. It should not be forgotten that even in the original gravity method, the distance friction coefficient $\beta$ remains still unsolved. Considering that the effectiveness of health service areas defined by the original gravity method is satisfied and that the data collection for the gravity extension model is hard, only the original gravity model was used in this paper. In future work, with the help of more data, a more effective model can be established for delimiting health service areas.

Second, we created the health service areas on the basis of a regional hospital's location both for the proximal area method and the gravity one. This raises the question that if these regional hospitals are distributed unevenly, their respective health service areas

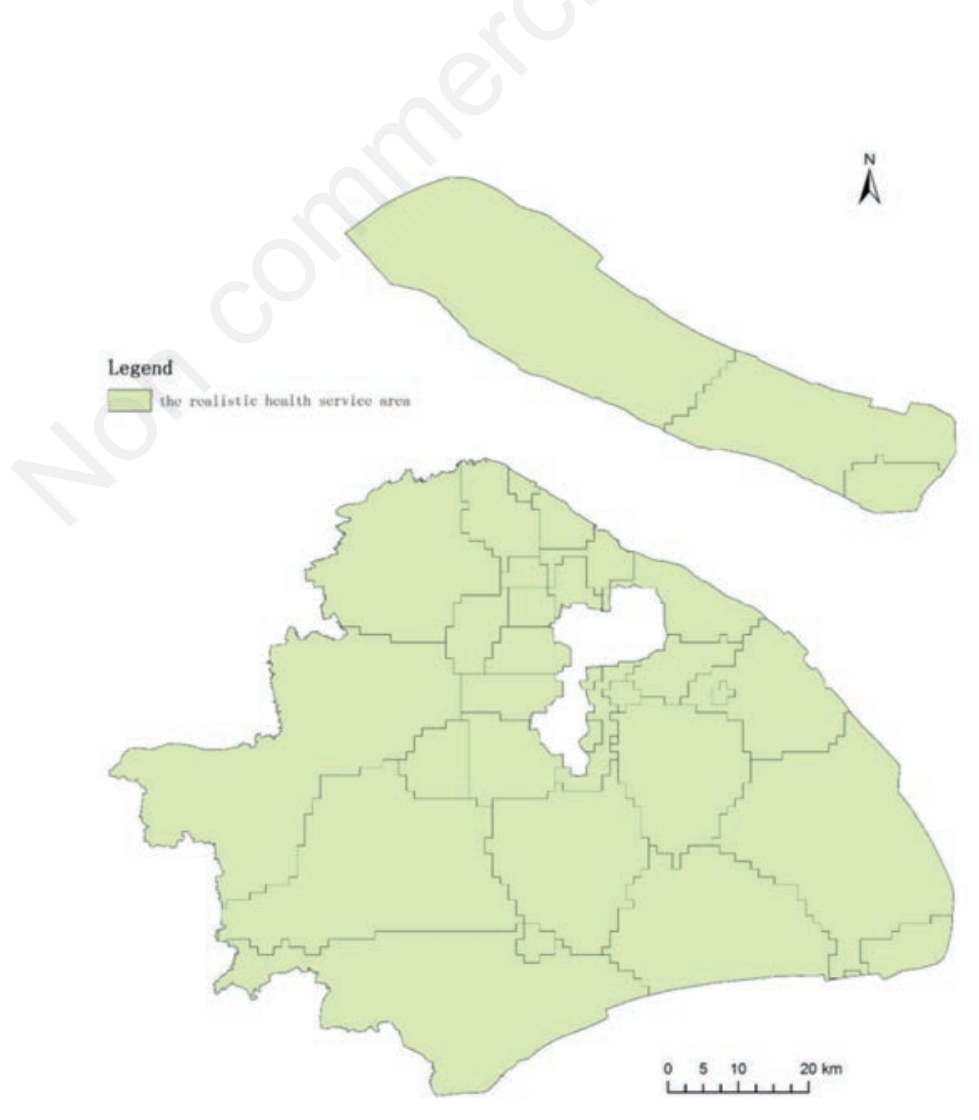

Figure 7. Realistic health service areas in Shanghai. 
will vary in size. Briefly, the spatial distribution of the regional hospitals chosen for this paper determines the range of health service areas. Therefore, it is important to make sure that the regional hospitals chosen for the creation of health service areas are relatively equal spatially, and that the scale of each regional hospital is similar.

\section{Limitations}

There is a large amount of data involved in this paper, including the spatial distributions of the population and hospitals, operational data of hospitals, administrative boundary data, road network data and so on, and this makes data collection difficult. In this paper, Shanghai, a municipality of China with 16 districts, is taken as an example city for delimiting health service areas. In the process of data collection, data from the Jingan District, Hongkou District, Yangpu District and Xuhui District are missing. Therefore, these four districts were excluded from the calculations and verification. It is well known that researchers need high-quality data, which can contribute to more accurate results. However, in reality, data collection is never perfect. In this paper, we aimed to find and confirm a method for delimiting health service areas in general, not delimit health service areas specifically for Shanghai. Therefore, we felt that the data, even though missing data from four districts, could be used for the purpose of this paper.

\section{Conclusions}

As it turns out, both the proximal method and the gravity method are suitable for delimiting health service areas in regional health planning. From a theoretical point of view the gravity method is superior to the simple proximal method, but the verification results show that the gravity method does not obviously surpass the proximal method in delimiting health service areas. In other words, given that the effectiveness of the result is the only factor under consideration, the proximal method and the gravity method provide similar results. However, seen another way, there are many differences between the two methods. In terms of the data used for modelling, spatial distribution data for the population and the hospitals, in addition to road network data are needed for the proximal method, while the gravity method also needs hospital operational data and the value of crucial parameter $\beta$, which is difficult to determine and therefore must be estimated. In terms of operation, the proximal method is easier to operate than the gravity method because the major calculation in the proximal method is determining the road network distance, while the calculation procedure of the gravity method, based on the road network distance, also adds the scale of the hospital and a distance friction coefficient to the calculation. This optimises calculating the gravity model but it also makes it difficult to intellectually grasp its complicated formula. However, although modelling based on the proximal method is simpler and easier to understand, it only considers distance in defining trade areas, while the gravity method considers two factors: the distance and the attractiveness of hospitals.

\section{References}

Applebaum W, 1965. Can store location research be a science. Econ Geog 41:234-7.
Applebaum W, 1966. Methods for determining store trade areas, market penetration and potential sales. J Mark Res 3:127-41.

Barber SL, 2014. The hospital of the future in China: China's reform of public hospitals and trends from industrialized countries. Health Policy Plann 29:367-78.

Berlin C, 2014. Avoidable hospitalizations in Switzerland: a small area analysis on regional variation, density of physicians, hospital supply and rurality. BMC Health Serv Res 14:289-99.

Dartmouth Institute for Policy and Clinical Practice, 1998. The quality of medical care in the United States: a report on the medicare program. The Dartmouth Atlas of Health Care, Chicago, IL, USA.

Garnick DW, Luft HS, Robinson JC, 1987. Appropriate measures of hospital market areas. Health Serv Res 22:69-89.

Erickson GM, Finkler SA. 1985. Determinants of market share of hospital's services. Med Care 23:1003-18.

Ge M, Jiang P, Zhao X-m, Xu L, Ma Z-1, Lu W, 2013. The institutional design to establish the regional medical association in Changning District, Shanghai. Chinese J Health Policy 12:1-9.

Ghosh A, McLafferty S, 1987. Location strategies for retail and service firms. DC Health, Lexington, MA, USA.

Guerrero EG, Kao D, 2013. Racial/ethnic minority and lowincome hotspots and their geographic proximity to integrated care providers. Subst Abuse Treat Prev Policy 8:34-44.

Hsiao WC, 1995. The Chinese health care system: lessons for other nations. Science 8:1047-55.

Huff DL, 1963. A probabilistic analysis of shopping center trade areas. Land Econ 39:81-90.

Huff DL, 1964. Defining and estimating a trading area. J Marketing 7:34-8.

Huff DL, 2003. Parameter estimation in the Huff model. Available from: https://www.esri.com/news/arcuser/1003/files/huff.pdf

Jin F, Wang F, Liu Y, 1980. Geographic patterns of air passenger transport in china 1980-98: imprints of economic growth, regional inequality and network development. Prof Geogr 56:471-87.

Klauss G, Staub L, Widmer M, Busato A, 2005. Hospital service areas: a new tool for health care planning in Switzerland. BMC Health Serv Res 5:33-48.

Leung GM, Wong IO, Chan WS, Choi S, Lo SV, Health Care Financing Study Group, 2005. The ecology of health care in Hong Kong. Soc Sci Med 61:577-90.

Nakanishi M, Cooper LG, 1974. Parameter estimates for multiplicative competitive interaction models: least square approach. J Mark Res 11:303-11.

National Development and Reform Commission, 2009. Opinions of the CPC Central Committee and the State Council on deepening the healthcare system reform. Available from: http://www.sda.gov.cn/WS01/CL0611/41193.html

Okabe A, Satoh T, Furuta T, 2008. Generalized network voronoi diagrams: concepts, computational methods, and applications. Int J Geogr Inf Sci 22:965-94.

Olsen LM, Lord JD, 1979. Market area characteristics and branch performance. J Bank Res 10:102-10.

Peipins LA, Graham S, Young R, 2013. Racial disparities in travel time to radiotherapy facilities in the Atlanta metropolitan area. Soc Sci Med 89:32-8.

Reilly WJ, 1931. The law of retail gravitation. Knickerbocker, New York, NY, USA.

Rongwen L, 2011. Establishment of three-level medical system based on two-way referral. China Acad J Electr 5:35-7. 
Shen X, 2013. Healthcare alliance through vertically integrating resources: an endeavor in Shanghai, China. China Acad J Electr 13:527-30.

Shi M, 2012. Status quo and institutional arrangement of primary health care system based on integrated perspective in China. Chinese J Health Policy 9:28-33.

Tao D, Chen Y, Wei X, 2012. Integrated healthcare system: International perspective and Chinese practices. Chinese $\mathrm{J}$ Health Policy 9:1-9.

Wang F, 2006. Quantitative methods and social-economic applications in GIS. CRC Press, New York, NY, USA.

Wang F, 2014. Quantitative methods and social-economic applications in GIS. CRC Press, New York, NY, USA.

White KL, 1997. The ecology of medical care: origins and implications for population-based healthcare research. Health Serv Res 32:11-21.
Yao P, 2015. Medical alliance's effect on promoting the accessibility of high-quality medical resources. Mod Hosp Manag 13:18-22.

Yip W, Hsiao WC, 2008. The Chinese health system at a cross roads. Health Aff 27:460-8.

Zhao Y, 2010. The type, source and strategy of difficulty and expensive access medical care under the situation of medical reform. Chinese Health Res 13:252-4.

Zhou J, 2009. Explore to construct regional medical consortium in shanghai: to enhance the efficiency in the use of health resources. Available from: http://en.cnki.com.cn/ Article_en/CJFDTOTAL-YZLT200906007.htm

Zhu F, 2014. Present situation and countermeasures of RuijinLuwan healthcare alliance in Shanghai. Chinese Hosp Manag $34: 21-3$. 